\title{
Negative mental representations in infancy
}

\author{
Jean-Rémy Hochmann ${ }^{1,2} *$, Juan M. Toro ${ }^{3,4}$ \\ Paper published in Cognition 2021
}

https://doi.org/10.1016/j.cognition.2021.104599

\footnotetext{
${ }^{1}$ CNRS UMR5229 - Institut des Sciences Cognitives Marc Jeannerod, 67 Boulevard Pinel, 69675, Bron, France.

${ }^{2}$ Université Lyon 1 Claude Bernard, France

${ }^{3}$ Institució Catalana de Recerca i Estudis Avançats, Pg. Lluis Companys, 23, 08019, Barcelona, Spain

${ }^{4}$ Universitat Pompeu Fabra, Roc Boronat, 138, 08018, Barcelona, Spain.
}

*Corresponding author: Jean-Rémy Hochmann; CNRS UMR5229, Institut des Sciences Cognitives Marc Jeannerod, 67 Boulevard Pinel, 69675, Bron, France. E-mail: hochmann@isc.cnrs.fr

\begin{abstract}
How do infants' thoughts compare to the thoughts adults express with language? In particular, can infants entertain negative representations, such as not red or not here? In four experiments, we used pupillometry to ask whether negative representations are possible without an external language. Eleven-month-olds were tested on their ability to detect and represent the abstract structure of sequences of syllables, defined by the relations identity and/or negation: AAAA (four identical syllables; Experiment 1), $\mathrm{AAA} \neg \mathrm{A}$ (three times the syllable $A$ and one final syllable that is not $A$; Experiment 2), $\mathrm{AA}(\mathrm{A})(\mathrm{A}) \neg \mathrm{A}$ (two-to-four times the syllable $A$ and one final syllable that is not A; Experiment 3). Representing the structures in Experiments 2-3 requires a form of negation. Results suggest that infants are able to compute both identity and negation. More generally, these results lend credit to the hypothesis that the infant mind is equipped with rudimentary logical operators before language takes off.
\end{abstract}


Pre-lexical negation in infancy

\section{Preamble}

\section{NAÎTRE HUMAIN}

In the early 1990s, Jacques Mehler and Emmanuel Dupoux (1990) published the book "Naître Humain" (To be born human; translated in English as “What Infants Know”). The title of Mehler and Dupoux's book has often made me wonder: how is "Naître Humain" different from "Naître Singe" (To be born a monkey) or "Naître Chimpazé"? Everyone agrees that human cognition eventually differs from other animals' cognition: only humans build huge towers, invent sophisticated tools like smart phones, communication systems like the Morse code, or external memories like books and the internet. What allows these achievements?

Inspired by the impressive achievements of current deep neural networks, as compared with connectionist networks in the 1990s, one could argue that the difference between humans and nonhumans lays in the computational power of our relatively large brain. If so, chimps, monkeys and humans would have the same core computations and representations of the world, but the greater computational power of the human brain would allow to develop those abilities further. This view predicts that all the important differences between humans and close species should develop gradually, as the brain matures and computational power increases.

Jacques Mehler (and others) challenged that view. He argued that human infants and other animals learn different things, because they have different "machineries", different brains and different cognitive systems. Throughout his career, Jacques advocated for the empirical study of those learning processes in human infants. There may be at least one fundamental ability present in humans that is not shared with other species. Chomsky (1995) and others (e.g. Hauser, Chomsky \& Fitch, 2002) have singled out the ability to combine mental representations, which would enable the productivity of human language and thought. Tomasello (2019) and others have emphasized the key role of joint attention in developing collaborations and enabling cultural transmission. The combinatoriality of the speech code, enabling the representation of an infinite 
number of words, may also be unique to humans (Liberman \& Mattingly, 1985; Pinker \& Jackendoff, 2005). Above and beyond the differences between those hypotheses, there is a shared view that human infants, in the earliest stages of development, may already exhibit certain human-unique abilities. The study reported below was inspired by Jacques's quest to understand human nature and identify precursors of human-unique cognitive abilities in young infants. Here, we focus on negation.

\section{INTRODUCTION}

Negation is a cognitive tool that relates truth and falsehood and is indispensable to allow human-unique achievements, such as logical reasoning and science (Horn, 1989). The ability to negate is typically appreciated through language (Papeo, Hochmann \& Batelli, 2016), so that it is unclear whether other species, which lack the human language faculty, and young children and infants, whose language faculty is immature, have negation. Children do not produce utterances involving logical negation before the third year of life (Choi, 1988; Dimroth, 2010; Feiman et al., 2017; Pea, 1980), but the hypothesis of a pre-lexical negation could account for infants' amazing learning abilities and early reasoning skills (Cesana-Arlotti et al., 2018). Here, we sought to advance on this debate, by investigating infants' ability to learn abstract relational structures that require a precursor of logical negation.

Though infants begin to say "no!" around their first birthday, it is generally accepted that this word expresses refusal or rejection of a contextual object (e.g., soup) or action, rather than logical negation (Choi, 1988; Dimroth, 2010; Feiman et al., 2017). Early meanings of "no" lack a defining feature of logical negation, that is, the expression of the relation between two contradictory mental representations (Hochmann, 2020; Pea, 1980): the current state of the world (the truth), and what it could have been, but is not (falsehood). When an infant refuse to eat soup, she is refusing an action (to eat) or an object (the soup), but she is not representing and asking for another type of food (reject soup does not define a type of food) or another action with soup 
(reject eating does not define an action). The object or action that is subjected to refusal/rejection is not put in relation to the mental representation of a different object or action.

Instead, logical negation entails a relation whereby a mental representation is defined in contrast to another mental representation. For instance, the proposition "the color of the ball is not red" expresses a negative relation between the actual color of the ball and the color red. The proposition essentially states that the ball has a color other than red (Pap, 1960). In a situation where the space of possibilities is well defined, like the definition of a color, negation entails the construction of the complement: computing not-x requires representing the ensemble to which $\mathrm{x}$ belongs, excluding x (e.g., all colors but red). Demonstrating that pre-verbal infants have a negation with all the properties of truth-functional negation is beyond the scope of the present study. In particular, it would require first to be able to identify propositional thoughts bearing truth values in infants. Rather, the experiments reported below aim at testing infants' ability to compute a precursor of negation that instantiates a negative relation between two mental representations, equivalent to the computation of the complement. If applied to propositional thoughts, such computation would be equivalent to logical negation.

There are reasons to doubt, as of today, that infants can compute such negation. Infants fail to represent negative rules (Benavides-Varela \& Mehler, 2015) or negative goals (Feiman, Carey \& Cushman, 2015). Relevant to this issue, are studies of pairs of contradictory concepts, where one concept is the negation of the other and no third option is possible (e.g., present/absent, same/different; Horn, 2018). Overall, as we will detail below, the results of these studies can be explained by attributing only the positive representations (present, same) to infants.

Take the case of present and absent. Many experiments have studied infants' reactions to the presence or absence of an object at a hiding location (e.g., behind a screen or inside a container), manipulating the evidence that the object, initially hidden, was eventually left at or removed from the hiding location (e.g., McCurry, Wilcox \& Wood, 2009; Wang, Baillargeon \& 
Paterson, 2005; Wilcox \& Baillargeon, 1998; Wilcox \& Schweinle, 2002). These studies show strong evidence that young infants have object permanence; i.e. they can represent the presence of an object, even when it is not immediately perceivable. They are thus surprised when the object is not found in the hiding location. In contrast, there is no evidence that infants can represent the absence of an object without the immediate perception of that absence. Indeed, when evidence was given for the removal of an object, but that object was eventually found, infants did not exhibit surprise (Kaufman, Csibra \& Johnson, 2003; Wynn \& Chiang, 1998). Infants could not represent and anticipate that the object would not be in a certain location.

A parallel pattern of results is observed with respect to infants' representations of same and different. Infants can perceive that two stimuli are the same or that two stimuli are different. In fact, the field of infant cognition relies heavily on such perception of sameness and difference in habituation-dishabituation studies (e.g., Eimas et al., 1971; Mehler et al., 1988). However, showing that infants can represent the abstract relations same and different, independently from what stimuli are the same or different, requires a generalization test. When it comes to abstract relations, there is strong evidence that infants can represent the relation same, but no evidence that they can represent the relation different (Hochmann et al., 2011, 2018; Hochmann, 2010; Hochmann, Carey \& Mehler, 2018; Kovács, 2014). Indeed, young infants discriminate exemplars of the concept same (e.g., two identical squares) and exemplars of the concept different (e.g., a square and a triangle) (Addyman \& Mareschal, 2010; Anderson, et al., 2018; Ferry, Hespos \& Gentner, 2015; Tyrrell et al., 1991; Walker \& Gopnik, 2014), but such discrimination likely only relies on a representation of same. In a critical study, infants were presented with varying triplets of stimuli instantiating both the relation same and the relation different (e.g. A A B; C D D; etc), and were trained either to systematically choose one of the stimuli following the relation same (A and $\mathrm{D}$ in the examples above) or to systematically choose following the relation different (B and $\mathrm{C}$ in the examples above). Although infants performed correctly in both conditions, subsequent 
tests revealed that their choice was always based on a representation of same: they learned to choose same, when that was the correct rule, and to avoid same, when the correct rule was to choose different (Hochmann, Mody \& Carey, 2016; see also Zentall et al., 1981; 2018 for convergent results with pigeons). Thus, as of today, there is no evidence that infants represent different, only evidence that they represent same (Hochmann, 2021).

In sum, the extant literature is compatible with the view that logical negation, or a precursor thereof that instantiates a negative relation between two mental representations, is absent in infants and may await maturation and/or linguistic competence to develop (Feiman et al., 2017). Nevertheless, even in adults, negation is not always recruited spontaneously (Beltrán, Orenes \& Santamaría, 2008). In particular, the efficient processing of a negative utterance requires the positive counterpart to be well represented and pragmatically relevant (Papeo \& de Vega, 2020; Wason, 1965). Analogously, if infants do not demonstrate the use of negative concepts such as absent (not present) or different (not the same), it may be because it is sufficient and easier to make sense of scenes and events with their positive counterparts. Moreover, even if logical negation were to emerge later in development, it cannot be created ex-nihilo. Some precursors of logical negation may therefore be observable in infancy.

The new paradigm developed in the current study has been conceived to assess a latent ability to instantiate a negative relation between two mental representations in infancy. We asked whether infants are able to generalize the common abstract structure of sequences, in which one element of each sequence is defined as the negation of another; e.g., $\mathrm{AAA} \neg \mathrm{A}$, where $\mathrm{A}$ is a variable for the elements composing the sequences. The first element $\mathrm{A}$ is repeated several times, so to emphasize its relevance. The final element is defined in a negative relation to the previous elements $(\neg \mathrm{A})$; it can take any value, but the value of A. We used speech stimuli (i.e., syllables), which are well represented, discriminated and categorized by the end of the first year of life (Hochmann \& Papeo, 2014; Werker \& Tees, 1984). We thus tested whether infants could detect 
and generalize structures of syllable sequences based on identity and negation (AAA $\neg$ A; e.g., $b a$ ba ba di, fu fu fu le, mi mi mi na).

We know that infants can detect and generalize abstract structures such as $\mathrm{AAB}$ or $\mathrm{ABA}$, where $\mathrm{A}$ and $\mathrm{B}$ are variables for syllables (AAB: pupuki, lelega, fofose, etc.; $\mathrm{ABA}$ : pukipu, legale, fosefo, etc.), in a way that allows them to discriminate between those structures (Kovács \& Mehler, 2009; Marcus et al., 1999). Similar results have been reported using non-verbal sounds, visual shapes or photographs (see Rabagliati et al., 2018 for a review). However, there is no evidence, so far, that B is represented as $\neg \mathrm{A}$. Representing the identity relation is in fact sufficient to discriminate between the structures $\mathrm{AAB}$ and $\mathrm{ABA}$ : $\mathrm{AAB}$ is defined by the identity of the first two elements; whereas $\mathrm{ABA}$ is defined by the identity of the first and last elements. The third element, which could be represented in a negative relation with A is so far irrelevant, or at least unnecessary, for discriminating between $\mathrm{AAB}$ and $\mathrm{ABA}$.

To show that infants indeed represent the element $\mathrm{B}$ as $\neg \mathrm{A}$, it should be found that infants expect the values of B and A to differ. Thus, we need to show that infants can discriminate the structure $\mathrm{AAB}$ from the structure $\mathrm{AAA}$, or $\mathrm{AAAB}$ from $\mathrm{AAAA}^{1}$. If infants consider a sequence such as $b a b a b a b a$ unfit for the AAAB structure, this would demonstrate their ability to expect the final syllable to be anything but $b a$ and their ability to represent the structure AAA $\neg \mathrm{A}$ relying on negation.

We applied this rationale in the experiments reported below, in which we measured the pupil dilation of 11-month-olds during an oddball paradigm. Crucially, 11-month-olds are still a year away from producing or understanding sentences containing logical negation (Feiman et al., 2017). In three experiments, infants were exposed to series of structured sequences of syllables. The syllables varied from one trial to another (48 different syllables were used for every infant), but they always formed sequences that matched either a frequent standard structure $(75 \%$ of trials) or an infrequent deviant structure (25\% of trials; Figure 1). If infants could detect the 
common abstract structure of standard sequences, they should react to violations of that structure (i.e., when the final syllable deviates from the expected one in deviant sequences). We used pupil dilation to measure the response to deviant stimuli (Hochmann, 2013; Hochmann \& Papeo, 2014). Increase in arousal, attention or cognitive load triggers an increase of pupil diameters (Beatty \& Kahneman, 1966; Hess \& Polt, 1960; Laeng, Sirois \& Gredebäck, 2012). In particular, in oddball paradigms, pupils dilate in reaction to unexpected auditory stimuli in both adults (Qiyuan et al., 1985; Quirins et al., 2018) and infants (Hochmann \& Papeo, 2014). Pupillometry thus provides a solid method to assess infants' cognition.

\section{Experiment 1 Experiment 2}

$75 \% \quad 25 \%$

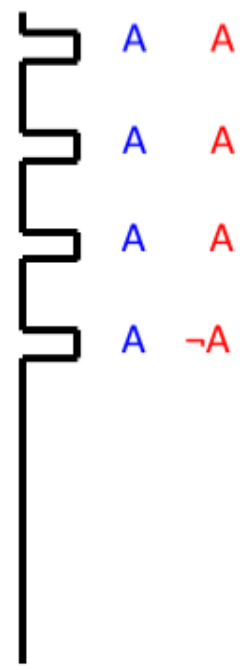

$75 \% \quad 25 \%$

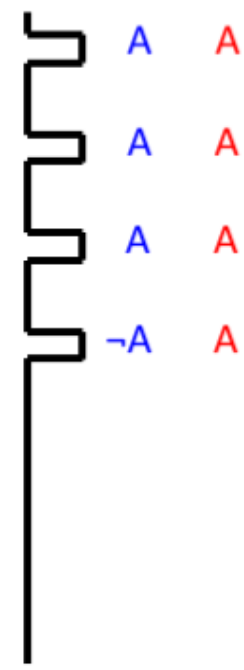

\section{Experiment 3}

$25 \% 25 \% 25 \% \quad 25 \%$

Figure 1 - Design of Experiments 1-3. $A$ and $\neg A$ could be any of 48 different syllables. Standard sequences are depicted in blue; deviant sequences are depicted in red.

In Experiment 1, we validated pupillometry as a tool to study structure generalization in infants, testing their representation of the structure AAAA that relies only on identity. In Experiments 2-3, we assessed infants' ability to learn a structure requiring negation (Figure 1).

\section{EXPERIMENT 1}

In Experiment 1, 11-month-old infants were tested on their ability to represent the AAAA structure relying only on identity relations. If infants represent the structure of standard AAAA 
sequences, we should observe pupil dilation in response to rare deviant AAAB sequences. This experiment mainly served as a validation of our methods.

\section{Methods}

\section{Participants}

Sixteen infants (age range: $10 \mathrm{~m} 2 \mathrm{~d}-12 \mathrm{~m} 15 \mathrm{~d}$; average: $11 \mathrm{~m} 1 \mathrm{~d}$ ) participated in Experiment 1. Four additional infants were tested but excluded for not providing a sufficient number of trials (see Analysis). The sample size was chosen in reference to previous work with a similar paradigm (Hochmann \& Papeo, 2014). All participants in Experiments 1-3a were recruited shortly after birth in Barcelona hospitals and tested in the babylab of Universitat Pompeu Fabra, Barcelona. These studies were approved by the "Parc de Salut MAR - Clinical Research Ethics Committee". Parents gave an informed consent before participating in the study and were informed that they could withdraw their consent at any time without any consequence.

\section{Stimuli}

Forty-eight syllables were created with the artificial speech synthesizer MBROLA (French voice database FR4), with phoneme duration of $120 \mathrm{~ms}$ and pitch of $200 \mathrm{~Hz}$. We used 12 consonants $(/ \mathrm{b} /, / \mathrm{d} /, / \mathrm{g} /, / \mathrm{p} /, / \mathrm{t} /, / \mathrm{k} /, / \mathrm{v} /, / \mathrm{f} /, / \mathrm{s} /, / \mathrm{l} /, / \mathrm{m} /, / \mathrm{n} /)$ and 4 vowels $(/ \mathrm{a} /, / \mathrm{i} /, / \mathrm{o} /, / \mathrm{u} /)$. Each syllable was normalized to an intensity of $70 \mathrm{~dB}$. These syllables were used to create standard sequences of four identical syllables (AAAA; e.g., $b a b a b a b a$, di di di di, $k u k u k u k u$, etc.) and deviant sequences of three identical and one different syllables (AAA $\neg$ A ; e.g., fi fi fi lo). The video shown repeatedly on the screen of the eyetracker consisted in an animated video clip showing a smiling cartoon character jumping repeatedly (www.GoAnimate.com).

\section{Procedure}

Infants sat on their parent's laps in front of a Tobii eyetracker T60XL. The presentation of stimuli and the recording of eye-tracking data at $60 \mathrm{~Hz}$ was controlled by PsyScope $\mathrm{X}$ (http://psy.cns.sissa.it). Parents' ears were covered, so that they could not hear the stimuli, and 
their eyes were close during the whole experimental session, so not to interfere with the eyetracker recordings. All lights in the room were switched off, except for those coming from the eyetracker screen. Each trial started automatically when infants fixated a central blinking cross presented on the screen. The central blinking cross disappeared and the jumping character appeared at the center of the screen. The pupil size is particularly sensitive to variations of luminance. To ensure that any observed effect could not be attributed to variations of luminance, contrast or any other visual features of the stimuli, we used the exact same video in all trials.

In each trial, a standard AAAA ( $75 \%$ of trials) or a deviant AAA $\neg \mathrm{A}(25 \%$ of trials) sequence was played by loudspeakers located behind the screen. The first syllable of each trial began $200 \mathrm{~ms}$ after the onset of the video clip. The onsets of two successive syllables were separated by $900 \mathrm{~ms}$. Each trial ended $2283 \mathrm{~ms}$ after the onset of the fourth syllable. In the first 8 trials of each experiment, only standard sequences were presented (AAAA). The experiment lasted until 96 additional trials were run (72 standard and 24 deviant trials), until the infant fussed out or until the parent asked to stop the experiment. Two trials were separated by a black screen displaying only a central blinking cross to attract infants' gaze. Trials were run in a pseudorandom order, so that two deviant trials were separated by 1 to 6 standard trials.

Analysis

Fixations were identified by PsyScope X following the dwell-time algorithm (Duchowski, 2007) with the following parameters: WindowLength $=200$, MinFixationLength $=100$, DistanceFromMean $=0.05$. We defined an area of interest (660 pi $x 432$ pi) corresponding to the surface of the video played on the screen to attract infants' gaze. The pupil diameter for both eyes was recorded for fixations in that area of interest. For each trial, we considered a baseline time window beginning $500 \mathrm{~ms}$ before the onset of the fourth syllable (time 0 ) of the sequence. The average pupil diameter in the baseline window was subtracted from all data points. 
To analyze pupil dilation, we need first to select trials that provide data in the time window where an effect can be expected. In previous studies using our oddball paradigm, we found that pupil dilation in response to a change detection lasts about $500 \mathrm{~ms}$ and peaks around $1500 \mathrm{~ms}$ after the onset of the relevant stimulus (Hochmann \& Papeo, 2014). In the extant literature relying on pupillometry in infants, this stands as an early effect (Sirois \& Jackson, 2012; Gredebäck \& Melinder, 2010; Geangu, Hauf, Bhardwaj, \& Bentz, 2011; Tamasi et al., 2017). An effect is unlikely to be observed before $1250 \mathrm{~ms}$. In consequence, we consider a time window of interest, beginning $1250 \mathrm{~ms}$ after the onset of the last syllable of each sequence and ending with the end of the trial $(2283 \mathrm{~ms})$.

In all experiments, the 8 first trials (all standard trials) constituted the familiarization to the standard structure. These trials are not included in the reported analysis, though results would be unchanged if they were to be included. In addition, we excluded trials with less than $100 \mathrm{~ms}$ of pupil diameter information during the baseline window $(-500-0 \mathrm{~ms})$ or less than $50 \%(516 \mathrm{~ms})$ of pupil diameter information in the window of interest. Infants with fewer than 2 good trials per Trial Type (Standard, Deviant) were excluded from further analyses. Missing data (due to eye blink or fixation out of the area of interest) for good trials were linearly interpolated. Table 1 summarizes the average number of trials included in the final analysis for each experiment.

Table 1 - Average number of trials included in the final analysis

\begin{tabular}{|c|c|c|c|c|}
\hline Experiment & Total \#trials & \#good trials $(\%)$ & \#good standard (\%) & \#good deviant (\%) \\
\hline Experiment 1 & 72.44 & $45.06(61 \%)$ & $33.12(60 \%)$ & $11.94(68 \%)$ \\
\hline Experiment 2 & 78.25 & $44.44(56 \%)$ & $33.38(55 \%)$ & $11.06(57 \%)$ \\
\hline Experiment $3 a^{*}$ & 33 & $17.56(51 \%)$ & $8.44(49 \%)$ & $9.12(53 \%)$ \\
\hline Experiment $3 b^{*}$ & 23.56 & $12(49 \%)$ & $5.94(49 \%)$ & $6.06(50 \%)$ \\
\hline
\end{tabular}


standard sequences. In addition, a non-parametrical cluster mass permutation test (Hochmann \& Papeo, 2014; Maris \& Oostenveld, 2007) was implemented to find significant pupil dilation in response to deviant trials compared to the standard trials, in the time window of interest. For each time point in that time window, we computed the $t$ statistics of the paired t-test comparing participants' pupil diameter variation in Deviant and Standard trials. The $t$-values of adjacent points above a threshold $(\mathrm{t}=1.753$ corresponding to $P<.051$-tail $)$ were added to define the cluster statistics. One thousand permutations were then run to assess the probability of finding clusters with higher statistics if the standard and deviant labels were assigned randomly.

\section{Results}

In Experiment 1, we validated the current experimental paradigm by testing the detection and generalization of a simple structure based on identity relations. Eleven-month-old infants were exposed to the standard structure AAAA, namely, sequences of four identical syllables. We observed significant pupil dilation in response to deviant $\mathrm{AAA} \neg \mathrm{A}$ sequences compared to standard sequences between 1250 and $1750 \mathrm{~ms}(\mathrm{t}(15)=2.97 ; P<.01 ; d=.74)$. In addition, a cluster mass permutation test revealed larger pupil dilation in response to infrequent deviant sequences (AAA $\neg \mathrm{A}$ ), as compared with standard sequences (AAAA), around 1500 ms (interval $1250-1767 \mathrm{~ms} ; P=.02$; Figure 2A). This demonstrated that infants expected the last syllable to be identical to the preceding ones and detected the violations of that rule.

Now that we validated our experimental paradigm, we return to the main issue of this paper, whether infants can represent a negative relation between two elements. In Experiment 2, we thus asked whether infants could represent the AAA $\neg \mathrm{A}$ structure, where the last syllable is constrained to differ from the preceding ones. 

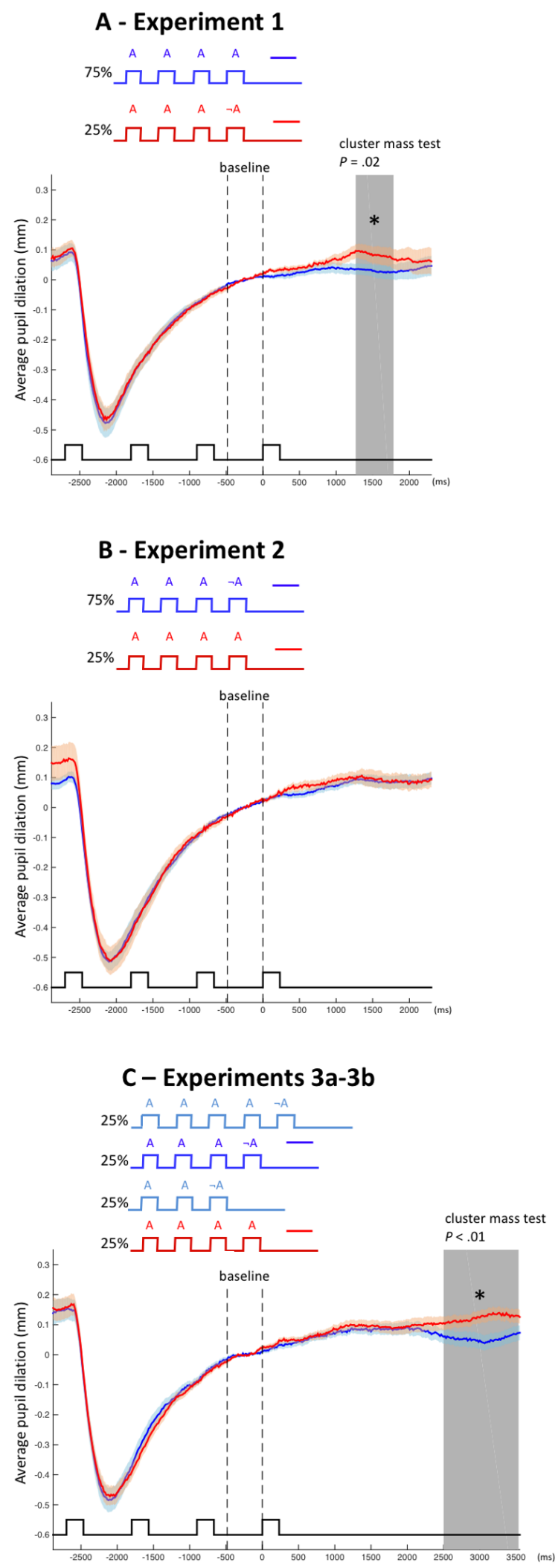

Figure 2 - Results of the pupil dilation measures in Experiments 1-3. The curves represent the average variations of pupil diameters $(\mathrm{mm})$ with respect to a $500 \mathrm{~ms}$ baseline taken before the onset of the $4^{\text {th }}$ syllable. The baseline is marked by two dotted vertical lines. Grey areas depict the time windows where a cluster mass permutation test detected significantly $(* P<.05)$ larger pupil dilation for deviant sequences $($ red) with respect to standard sequences (blue). Light-colored areas represent standard errors from the mean. The crenel functions indicate when syllables occurred within each trial. 


\section{EXPERIMENT 2}

In Experiment 2, 11-month-old infants were tested on their ability to represent the AAA $\neg \mathrm{A}$ structure (i.e., three times the syllable A and one final syllable that is not A) relying on both identity and negation. If infants represent the structure of standard AAAB sequences, we should observe pupil dilation in response to rare deviant AAAA sequences.

\section{Methods}

The procedure and analyses were the same as in Experiment 1, except that standard sequences now followed the AAA $\neg \mathrm{A}$ structure $(75 \%$ of trials) and deviant sequences followed the AAAA structure $(25 \%$ of trials $)$.

\section{Participants}

Sixteen infants (age range: $9 \mathrm{~m} 16 \mathrm{~d}-12 \mathrm{~m} 14 \mathrm{~d}$; average: $11 \mathrm{~m} 12 \mathrm{~d}$ ) participated in Experiment

2. Fifteen ${ }^{2}$ additional infants were tested but excluded for not providing a sufficient number of trials (see Analysis).

\section{Results}

In Experiment 2, 11-month-old infants were exposed to the standard structure AAA $\neg \mathrm{A}$ involving both the identity relation (the first three syllables are identical: A) and negation (the last syllable is not A). No difference in pupil dilation in response to deviant (AAAA) and standard sequences were observed between 1250 and $1750 \mathrm{~ms}(\mathrm{t}(15)=.25 ; P=.80 ; d=.06)$. Moreover, a cluster mass permutation test failed to identify any significant change in pupil dilation in response to the deviant sequences AAAA that violated the structure of standard sequences (Figure 2B). These results indicate that, after hearing for instance $b a b a b a$, infants were not surprised to hear $b a$ again. Thus, infants did not expect the last syllable to be not ba. They did not compute negation.

However, this failure may be circumstantial. Representing the structure AAA $\neg$ A requires representing three identical elements plus the final negative relation. Representing three identical 
elements already severely taps the limited capacity of infants' working memory (Feigenson \& Carey, 2005), so that infants may not have sufficient cognitive resources left to add the representation of $\neg \mathrm{A}$. Moreover, a recent study showed that the integration of numerical information and a relational structure is a complex process that involves language-related neural resources in human adults (Wang et al., 2015). Infants may succeed at integrating numerical information (i.e., the number of syllables) with a relatively simple relational structure based only on identity (Experiment 1), but not with a more complex structure that involves both identity and negation (Experiment 2). Thus, Experiment 3 was designed to prevent the computation of numerical information, freeing resources for the representation of the final negative relation.

\section{EXPERIMENTS 3a-3b}

In Experiment 3a and its replication Experiment 3b, 11-month-old infants were exposed to a standard structure similar to that of Experiment 2, except for the number of identical syllables preceding the final different one, which varied between 2 and $4(\mathrm{AA} \neg \mathrm{A}, 25 \%$; $\mathrm{AAA} \neg \mathrm{A}, 25 \%$ or AAAA $\neg$ A, 25\%; Figure 1). This made numerical information irrelevant, as it was varying constantly. The standard sequences could rather be represented as an undefined number of identical syllables followed by a final different syllable $(\mathrm{AA}(\mathrm{A})(\mathrm{A}) \neg \mathrm{A})$. We tested for infants' ability to represent the final syllable as $\neg \mathrm{A}$ by presenting deviant sequences consisting in four identical syllables (AAAA, 25\%). Since infants could not anticipate the exact position of the final different syllable (i.e., after how many syllables it would occur), we predicted a delay in the pupil response to the deviant trials missing the final different syllable (AAAA). After the presentation of the fourth identical syllable, infants would have to wait at least one inter-syllable interval (ISI $=$ $900 \mathrm{~ms}$ ), before realizing that the sequence had ended and that the standard structure had been violated by the absence of the final different syllable. 


\section{Methods}

The procedure and analyses of Experiment 3a were similar to those of Experiments 1-2, except for the differences listed below. Experiment $3 b$ is a replication of Experiment $3 a$ in a different laboratory with a doubled sample size.

\section{Participants}

Sixteen infants (age range: $10 \mathrm{~m} 13 \mathrm{~d}-12 \mathrm{~m} 24 \mathrm{~d}$; average: $11 \mathrm{~m} 24 \mathrm{~d}$ ) participated in Experiment 3a. Eleven additional infants were tested but excluded for not providing a sufficient number of trials (see Analysis).

Thirty-two infants (age range: $10 \mathrm{~m} 0 \mathrm{~d}-12 \mathrm{~m} 7 \mathrm{~d}$; average: $11 \mathrm{~m} 0 \mathrm{~d}$ ) participated in Experiment 3b. Twenty-two additional infants were tested but excluded for not providing a sufficient number of trials. All participants were recruited through the consultation of birth records at the city halls. Infants were tested in the babylab of Institut des Sciences Cognitives Marc Jeannerod in Bron, France. This study was approved by the "Comité de Protection des Personnes Sud Est II". Parents gave an informed consent before participating in the study and were informed that they could withdraw their consent at any time without any consequence.

\section{Procedure}

The procedure of Experiments 3a-3b was identical to that of Experiments 1-2, except for two aspects. First, the standard sequences consisted of 2 to 4 identical syllables followed by a final different syllable. The deviant sequences consisted of exactly 4 identical syllables. This resulted in four equiprobable types of sequences; standard: $\mathrm{AA} \neg \mathrm{A}(25 \%), \mathrm{AAA} \neg \mathrm{A}(25 \%)$, AAAA $\neg$ A $(25 \%)$; deviant: AAAA $(25 \%)$. Second, the time following the last syllable was increased to $3533 \mathrm{~ms}$, as we expected pupil dilation to be delayed by at least $900 \mathrm{~ms}$, the intersyllable interval (see below). 
Analysis - Experiments $3 a-3 b$

The Analysis of Experiments 3a-3b differed from that of Experiments 1-2 in only one aspect. Because the number of syllables varied in Experiments 3a-3b, infants could not know in advance which syllable would be final and therefore different. In fact, a sequence of four identical syllables was legal as long as it was followed by a fifth different syllable (AAAA $\neg$ A). Thus, when presented with an AAAA sequence, infants could only know a posteriori that this sequence did not respect the standard structure, once they realized the sequence was over. We thus predicted that pupil dilation in response to deviant trials, if it occured, should be delayed by at least $900 \mathrm{~ms}$, the inter-syllable interval, compared to Experiments 1-2. In Experiments 3a-3b, we thus ran a planned t-test comparing average pupil dilation in the $2150-2650 \mathrm{~ms}(1250-1750+900 \mathrm{~ms})$ time window in response to deviant and standard sequences and ran a mass cluster permutation test in a time window of interest, beginning $2150 \mathrm{~ms}(1250+900 \mathrm{~ms})$ after the onset of the fourth syllable of each sequence and ending with the end of the trial (3533 ms).

In addition, because concurrent audio-visual stimulation attracts infants' attention, which in turn impacts pupil diameters, only those trials with the same number of syllables are comparable. We thus only compared standard $\mathrm{AAA} \neg \mathrm{A}$ and deviant AAAA trials. Note that those are the same sequences used in Experiments 1-2. AA $\neg \mathrm{A}$ and $\mathrm{AAAA} \neg \mathrm{A}$ sequences were not analyzed. The rest of the analysis was the same as for Experiments 1-2.

\section{Results}

\section{Experiment $3 a$}

No differences in pupil dilation in response to deviant and standard sequences were observed between 2150 and $2650 \mathrm{~ms}(\mathrm{t}(15)=1.23 ; P=.24 ; d=.31)$. However, a cluster mass permutation test found larger pupil dilation in response to deviant sequences AAAA, as compared with standard sequences of same length $(\mathrm{AAA} \neg \mathrm{A})(P=.02)$. This effect occurred around $2900 \mathrm{~ms}$ (interval 2533-3283 ms), that is $1400 \mathrm{~ms}$ later than the pupil dilation observed in Experiment 1. 
This shows that, after hearing the same syllable A repeated a number of times, 11-month-old infants expected the final syllable to be anything but A. Thus, infants can represent the structure $\mathrm{AA}(\mathrm{A})(\mathrm{A}) \neg \mathrm{A}$.

Experiment $3 b$

Experiment $3 \mathrm{~b}$ replicated the results of Experiment $3 \mathrm{a}$ in a different lab with a doubled sample size. Again, no differences in pupil dilation in response to deviant and standard sequences were observed between 2150 and $2650 \mathrm{~ms}(\mathrm{t}(31)=.98 ; P=.33 ; d=.17)$; but a cluster mass permutation test found significantly larger pupil dilation for deviant AAAA sequences, relative to standard AAA $\neg$ A sequences between 2933 and $3317 \mathrm{~ms}(P=.04)$.

\section{Experiments $3 a$ and $3 b$}

Combining the data of Experiments $3 \mathrm{a}$ and $3 \mathrm{~b}(\mathrm{~N}=48)$, no difference in pupil dilation in response to deviant and standard sequences were observed between 2150 and $2650 \mathrm{~ms}(\mathrm{t}(47)=$ $1.42 ; P=.16 ; d=.20)$. However, a cluster mass permutation test found significantly larger pupil dilation for deviant than for standard sequences between 2500 and $3534 \mathrm{~ms}(P<.01$, Figure $2 \mathrm{C})$. A second cluster mass permutation test was run to test for the interaction between the lab factor (Experiment 3a; Experiment 3b) and the Sequence Type (Standard, Deviant). No effect was observed.

\section{DISCUSSION}

We used pupillometry to probe infants' ability to represent abstract relational structures. Eleven-month-old infants successfully learned the identity-based structure (AAAA, Experiment 1), but initially failed to learn the structure based on both identity and negation (AAA $\neg \mathrm{A}$, Experiment 2). We hypothesized that this failure reflected limited cognitive resources and/or the difficulty of combining a complex relational structure entailing negation with the information about the number of identical elements in the sequence. In Experiments 3a-3b, numerical information was made irrelevant by varying the number of syllables in each sequence: two to four 
identical syllables preceded a final different one. Pupil dilation was observed in response to the deviant sequences of four identical syllables, lacking the final different syllable. Thus, by eliminating a competing process (i.e., tracking the number of identical items in a sequence), we demonstrated that 11-month-old infants are able to learn the abstract structure $\mathrm{AA}(\mathrm{A})(\mathrm{A}) \neg \mathrm{A}$ based on negation.

It is important to emphasize that a number of intuitive explanations cannot account for the results of Experiments 3a-3b. First, the pupil dilation that we observed is not a reaction to a local change detection or to the perception of a local difference (e.g., the change between the repeated syllable $b a$ and $d i$ ), as would happen in habituation-dishabituation paradigms. Indeed, pupil dilation is not observed in response to a change of syllable but in response to the absence of such change in AAAA sequences.

Second, our results cannot be explained by a representation of heterogeneity. Inspired by Shannon's information theory, entropy is a continuous measure of heterogeneity that has been proposed to constitute a precursor to the concepts same and different (Wasserman \& Young, 2010). A sequence of four different elements (ABCD) has an entropy of 2 , a sequence of identical elements (e.g., AAAA) has an entropy of 0 and a sequence of three identical and one different elements $(\mathrm{AAAB})$ has an entropy of $.81^{3}$. One could imagine that infants discriminate between homogeneous (entropy $=0$ ) and heterogeneous (entropy $>0$ ) sequences, but this is not the case. Young children in fact discriminate well between entropy 0 and 4 , but not between 0 and 1 , let alone between 0 and .81 (Hochmann et al., 2017). Therefore, entropy cannot account for the results observed in our experiments.

Third, our results cannot be accounted for by attributing only a representation of identity to infants. Sequences of identical syllables were not surprising per se, as 2 to 4 identical syllables occurred in every trial. Sequences of exactly four identical syllables even occurred in half of the trials (standard AAAA $\neg$ A $25 \%$; deviant AAAA 25\%). Moreover, the effect of violation was 
delayed by $1400 \mathrm{~ms}$ compared to Experiment 1. This delay is larger than the ISI of $900 \mathrm{~ms}$, which was the minimal time to discriminate between a deviant AAAA and a standard AAAA $\neg \mathrm{A}$ sequence. These observations thus show that infants did not react to a sequence of identical syllables, or to the fourth identical syllable, but rather reacted to the absence of an expected final different syllable.

In Experiments 3a-3b, infants thus formed a mental representation of the final syllable as necessarily different from the previous ones. They had specified a negative relation between the expected final syllable and the syllable initially repeated in the sequence. When hearing $b a b a$, infants could not predict whether the final syllable would be the third, fourth or fifth syllable. Neither could they predict what syllable would end the sequence (whether it would be $d i, k o, s u$ or else). But infants could predict that the last syllable would be not $b a$, and reacted to the violation of that prediction.

By changing the syllables in every trial, we further demonstrated infants' ability to generalize the above process: if syllable A defines the beginning of the sequence, the last syllable must be not $A$, above and beyond the particular identity of A. This shows that infants dispose of a precursor of logical negation that instantiates a negative relation between two mental representations and operates over an abstract variable. Here, the variable symbolizes the syllable, a perceptual object defined in the speech domain. Further empirical investigations should generalize our results to other modalities, define the domains in which infant negation can apply, and particularly whether it can apply to propositional representations.

In conclusion, we showed that 11-month-old infants are able to represent structures defined by the identity relation and a precursor of negation. These findings constitute the first evidence that the ability to establish a negative relationship between two mental representations, a cornerstone of human reasoning, is in place by the end of the first year of life. More generally, 
these results lend credit to the hypothesis that the infant mind is equipped with rudimentary logical operators before language takes off.

\section{Author Contributions}

JRH designed the studies, collected and analyzed the data. JRH and JMTS wrote the paper.

\section{Acknowledgements}

This work was funded by the Agence Nationale pour la Recherche grant ANR-16-CE28-0006TACTIC awarded to JRH, the H2020 European Research Council (ERC) Starting Grant agreement 312519 awarded to JMTS and the collaborative McDonnell Foundation Grant 220020449. We thank Auriane Couderc, Roser Nicolau, Cinta Bertomeu and Claire Colsoulle for their help in recruiting and testing babies; Liuba Papeo, Luca Bonatti, Judit Gervain, Roman Feiman, Susan Carey and Liuba Papeo for comments on previous versions of this manuscript. Finally, we would like to dedicate this work to the warm memory of our mentor Jacques Mehler.

\section{Footnotes}

1 - AAB and AAA could potentially be discriminated on the basis of the number of identical elements. AAA contains 3 identical elements, whereas AAB contains only 2. Infants can discriminate between 2 and 3 syllables (Bijeljac-Babic, Bertoncini \& Mehler, 1993). In contrast, assuming infants do not discriminate between 3 and 4 elements (Feigenson \& Carey, 2005), AAAB and AAAA could not be discriminated on the basis of the number of identical elements. Representing AAAB in a way that allows discrimination from AAAA would require representing that the last element is not $\mathrm{A}(\neg \mathrm{A})$.

2 - Note that the attrition rates varied between the different experiments. Twenty percent (4 out of 20) of tested infants were excluded from the final analysis in Experiment 1; almost 50\% (15 out 
of 31 ) in Experiment 2 and about 40\% (11 out of 27; 22 out of 54) in Experiments 3a and 3b.

Different factors could account for the relatively high attrition rates in Experiments 2 and 3a-3b.

In Experiment 2, the high attrition rate could thus tentatively be interpreted as an additional piece of evidence that infants failed to represent the AAAB structure: many infants may lose interest in our stimuli because they do not understand their structure.

In Experiment 3a and 3b, a different factor may explain the relatively high attrition rate. Because we predicted that the effect would be delayed compared to that observed in Experiment 1, we increased the duration of trials by increasing the silence period following the end of the sequences. The longer the trials, the more likely infants are to look away. This design lead us to reject more trials (Table 1) and consequently more infants for not contributing enough trials.

3 - Considering a sequence of $\mathrm{N}$ elements composed of $\mathrm{S}$ identical elements and D different elements, following Wasserman \& Young (2010), Entropy $=-\mathrm{D} / \mathrm{N} \log _{2}(1 / \mathrm{N})-\mathrm{S} / \mathrm{N} \log _{2}(\mathrm{~S} / \mathrm{N})$.

\section{References}

Addyman, C., \& Mareschal, D. (2010). The perceptual origins of the abstract same/different concept in human infants. Animal Cognition, 13(6), 817-833.

Anderson, E.M., Chang, Y., Hespos, S., \& Gentner, D. (2018). Comparison within pairs promotes analogical abstraction in three-month-olds. Cognition, 176, 74-86.

Beatty, J., Kahneman, D. (1966). Pupillary changes in two memory tasks. Psychonomic Science 5, $371-372$.

Beltrán, D., Orenes, I., \& Santamaría, C. (2008). Context effects on the spontaneous production of negation. Intercultural Pragmatics, 5(4), 409-419.

Benavides-Varela, S., \& Mehler, J. (2015). Verbal positional memory in 7-month-olds. Child development, 86(1), 209-223. 
Bijeljac-Babic, R., Bertoncini, J., \& Mehler, J. (1993). How do 4-day-old infants categorize multisyllabic utterances? Developmental psychology, 29(4), 711.

Carey, S. (2009). The origin of concepts. Oxford University Press.

Cesana-Arlotti, N., Martin, A., Téglás, E., Vorobyova, L., Cetnarski, R., \& Bonatti, L. L. (2018). Precursors of logical reasoning in preverbal human infants. Science, 359, 1263-1266.

Choi, S. (1988). The semantic development of negation: a cross-linguistic longitudinal study. Journal of child language, 15, 517-531.

Chomsky, N. (1995). The minimalist program. MIT press.

Dimroth, C. (2010). The acquisition of negation, The expression of negation, 39-73.

Duchowski, A. (2007). Eye tracking methodology: Theory and practice (Vol. 373). Springer Science \& Business Media.

Eimas, P. D., Siqueland, E. R., Jusczyk, P., \& Vigorito, J. (1971). Speech perception in infants. Science, 171(3968), 303-306.

Feigenson, L., \& Carey, S. (2005). On the limits of infants' quantification of small object arrays. Cognition, 97(3), 295-313.

Feiman, R., Carey, S., \& Cushman, F. (2015). Infants' representations of others' goals: Representing approach over avoidance. Cognition, 136, 204-214.

Feiman, R., Mody, S., Sanborn, S., \& Carey, S. (2017). What do you mean, no? Toddlers' comprehension of logical "no" and "not". Language Learning and Development, 13(4),

Ferry, A. L., Hespos, S. J., \& Gentner, D. (2015). Prelinguistic relational concepts: Investigating analogical processing in infants. Child Development, 86(5), 1386-1405.

Hess, E. H., \& Polt, J. M. (1960). Pupil size as related to interest value of visual stimuli. Science 132, 349-350.

Geangu, E., Hauf, P., Bhardwaj, R., \& Bentz, W. (2011). Infant pupil diameter changes in response to others' positive and negative emotions. PLoS ONE, 6(11), Article e27132 
Gredebäck, G., \& Melinder, A. (2010). Infants' understanding of everyday social interactions: A dual process account. Cognition, 114, 197-206.

Hauser, M. D., Chomsky, N., \& Fitch, W. T. (2002). The faculty of language: what is it, who has it, and how did it evolve? Science, 298(5598), 1569-1579.

Hochmann, J. R. (2010). Categories, words and rules in language acquisition. (Doctoral Dissertation) http://hdl.handle.net/20.500.11767/4139

Hochmann, J-R. (2013). Pupillometry in six-month-old infants. In Baiz, Sarah, Eleanor Goldman, and Rachel Hawkes (eds.), Proceedings of the 37th Annual Conference on Language Development. Cascadilla Press

Hochmann, J-R. (2020). Cognitive precursors of negation in pre-verbal infants. In V. Deprez \& M. T. Espinal (Eds.), The Oxford Handbook of Negation. Oxford University Press.

Hochmann, J-R. (2021). Asymmetry in the complexity of same and different representations.

\section{Current Opinion in Behavioral Sciences}

Hochmann, J-R., Benavides-Varela, S., Fló, A., Nespor, M., \& Mehler, J. (2018). Bias for vocalic over consonantal information in 6-month-olds. Infancy, 23, 136-151.

Hochmann, J-R., Benavides-Varela, S., Nespor, M., \& Mehler, J. (2011). Vowels and Consonants in Early Language Acquisition. Developmental Science, 14, 1445-1458.

Hochmann, J-R., Carey, S., \& Mehler, J. (2018). Infants learn a rule predicated on the relation same but fail to simultaneously learn a rule predicated on the relation different. Cognition 177, 49-57.

Hochmann, J-R., Mody, S., \& Carey, S. (2016). Infants' representations of same and different in match- and non-match-to sample. Cognitive Psychology 86, 87-111.

Hochmann, J-R., \& Papeo, L. (2014). The invariance problem in infancy: a pupillometry study. Psychological Science 25, 2038-2046. 
Hochmann, J. R., Tuerk, A. S., Sanborn, S., Zhu, R., Long, R., Dempster, M., \& Carey, S. (2017). Children's representation of abstract relations in relational/array match-to-sample tasks. Cognitive Psychology, 99, 17-43.

Horn, L. (1989), A Natural History of Negation. Chicago: University of Chicago Press. 430-450.

Horn, L., R. (2018). Contradiction, in The Stanford Encyclopedia of Philosophy (Winter 2018 Edition), Edward N. Zalta (ed.).

Kaufman, J., Csibra, G., \& Johnson, M. H. (2003). Representing occluded objects in the human infant brain. Proceedings of the Royal Society of London. Series B: Biological Sciences, 270(suppl_2), S140-S143.

Kovács, Á. M. (2014). Extracting regularities from noise: Do infants encode patterns based on same and different relations? Language Learning, 64(2), 65-85.

Kovács, Á. M., \& Mehler, J. (2009). Flexible learning of multiple speech structures in bilingual infants. science, 325(5940), 611-612.

Laeng, B., Sirois, S., \& Gredebäck, G. (2012). Pupillometry: a window to the preconscious? Perspectives on Psychological Science 7, 18-27.

Liberman, A. M., \& Mattingly, I. G. (1985). The motor theory of speech perception revised. Cognition, 21(1), 1-36.

Marcus, G. F., Vijayan, S., Bandi Rao, S., \& Vishton, P. M. (1999). Rule-learning in sevenmonth-old infants. Science 283, 77-80.

Maris, E., \& Oostenveld, R. (2007). Nonparametric statistical testing of EEG- and MEG-data. Journal of Neuroscience Methods 164, 177-190.

McCurry, S., Wilcox, T., \& Woods, R. (2009). Beyond the search barrier: A new task for assessing object individuation in young infants. Infant Behavior and Development, 32, $429-436$.

Mehler, J. \& Dupoux, E. (1990). Naître Humain, Paris: Odile Jacob. 
Mehler, J., Jusczyk, P., Lambertz, G., Halsted, N., Bertoncini, J., \& Amiel-Tison, C. (1988). A precursor of language acquisition in young infants. Cognition, 29(2), 143-178.

Pap, A. (1960). “Types and Meaninglessness.” Mind, 69, 41-54.

Papeo, L., \& de Vega, M. (2020). The neurobiology of lexical and sentential negation. In V. Deprez \& M. T. Espinal (Eds.), The Oxford Handbook of Negation. Oxford University Press.

Papeo, L., Hochmann, J-R., Battelli, L. (2016). The default computation of negated meanings. Journal of cognitive neuroscience 28 (12), 1980-1986.

Pea, R. D. (1980). Logic in early child language. Annals of the New York Academy of Sciences, $345,27-43$.

Pinker, S., \& Jackendoff, R. (2005). The faculty of language: What's special about it? Cognition, $95,201-236$.

Qiyuan, J., Richer, F., Wagoner,B. L., \& Beatty, J. (1985). The pupil and stimulus probability. Psychophysiology 22, 530-534.

Quirins, M., Marois, C., Valente, M., Seassau, M., Weiss, N., El Karoui, I., Hochmann, J-R., \& Naccache, L. (2018). Conscious processing of auditory regularities induces a pupil dilation. Scientific Reports 8, 14819.

Rabagliati, H., Ferguson, B., Lew-Williams, C. (2018). The profile of abstract rule learning in infancy: Meta-analytic and experimental evidence. Developmental Science, e12704.

Sirois, S., \& Jackson, I. R. (2012). Pupil dilation and object permanence in infants. Infancy, 17, $61-78$.

Tamási, K., McKean, C., Gafos, A., Fritzsche, T., \& Höhle, B. (2017). Pupillometry registers toddlers' sensitivity to degrees of mispronunciation. Journal of Experimental Child Psychology, 153, 140-148.

Tomasello, M. (2019). Becoming human: A theory of ontogeny. Belknap Press. 
Tyrrell, D. J., Stauffer, L. B., \& Snowman, L. G. (1991). Perception of abstract identity/difference relationships by infants. Infant Behavior and Development, 14(1), 125-129.

Walker, C. M., \& Gopnik, A. (2014). Toddlers infer higher-order relational principles in causal learning. Psychological Science, 24, 87-92.

Wang, S. H., Baillargeon, R., \& Paterson, S. (2005). Detecting continuity violations in infancy: A new account and new evidence from covering and tube events. Cognition, 95(2), 129-173.

Wason, P. C. (1965). The contexts of plausible denial. Journal of verbal learning and verbal behavior, 4(1), 7-11.

Wasserman, E. A., \& Young, M. E. (2010). Same-different discrimination: The keel and backbone of thought and reasoning. Journal of Experimental Psychology: Animal Behavior Processes, 36(1), 3.

Werker, J. F., \& Tees, R. C. (1984). Cross-language speech perception: Evidence for perceptual reorganization during the first year of life. Infant behavior and development, 7(1), 49-63.

Wilcox, T., Baillargeon, R. (1998). Object individuation in infancy: The use of featural information in reasoning about occlusion events. Cognitive Psychology, 37, 97-155.

Wilcox, T., \& Schweinle, A. (2002). Object individuation and event mapping: Developmental changes in infants' use of featural information. Developmental Science, 5, 132-150.

Wynn, K., \& Chiang, W. C. (1998). Limits to infants' knowledge of objects: the case of magical appearance. Psychological Science, 9(6), 448-455.

Xu, F., \& Baker, A. (2005). Object individuation in 10-month-old infants using a simplified manual search method. Journal of Cognition and Development, 6, 307-323.

Zentall, T. R., Andrews, D. M., \& Case, J. P. (2018). Sameness may be a natural concept that does not require learning. Psychological Science, 29(7), 1185-1189. 
Zentall, T. R., Edwards, C. A., Moore, B. S., \& Hogan, D. E. (1981). Identity: The basis for both matching and oddity learning in pigeons. Journal of Experimental Psychology: Animal Behavior Processes, 7(1), 70. 